\title{
Load balancing of heterogeneous parallel DC-DC converter
}

\author{
Samuel Žák \\ University of Žilina \\ Faculty of Management Science and Informatics, \\ Univerzitná 8215/1, Žilina 01026 \\ Email: samuel.zak@fri.uniza.sk
}

\author{
Jaroslav Szabo \\ University of Žilina \\ Faculty of Management Science and Informatics, \\ Univerzitná 8215/1, Žilina 01026 \\ Email: jaroslav.szabo@fri.uniza.sk
}

\begin{abstract}
Switching power converters can achieve very high efficiency. However, they do so only for slim subset of operating conditions. Power supplies and loads with highly variable parameters do not operate at peak performance all the time. Energy harvesting and battery powered systems are typical examples. This paper studies the convenience of heterogeneous parallel DC-DC converter as a solution to this problem. The use of multiple diverse converter topologies in parallel could expand range of operating conditions with high efficiency. Such solution poses another issue of balancing workload across all topologies in system with minimal computational power. Efficiency of proposed converter is estimated by modelling converter circuits and running heuristic optimization on these models.
\end{abstract}

\section{INTRODUCTION}

$\mathbf{E}$ FFICIENCY of DC-DC converters can exceed $90 \%$ for specific operating conditions. If power supply is stable and load operates at constant voltage converter design can be easily optimized. For example when powering lowvoltage electronics from high-voltage grid. Battery powered devices complicate circuit optimization as voltage of the battery changes in relation to its capacity. Use of energy harvesting presents further complications due to wide range of operating voltage, available power and their variability over time. Maximum power point tracking methods can adapt operating point of the converter to extract the most from the supply. The limit of such adaptation is in the circuit design. Aim of this paper is to study the use of DC-DC converter consisting of multiple parallel lines with variable topologies. High variance of these topologies is important, so each parallel branch reaches peak performance at different operating conditions. This brings another problem, how to divide required output current among available topologies. It is important to note, that the power consumption of the converter itself needs to be minimal, as it is also considered power loss which decreases the overall efficiency of the power management system. Required computation of the balancing can use limited resources of low-power microcontroller or has to be performed elsewhere. To test both methods, we first create mathematical model of power loss of selected topologies, where brute-force algorithm running on computer with high computational power can find optimal operating distribution for given precision. Afterwards we used created models to develop heuristic optimization algorithm, capable of running on device with limited computational power. Proposed converter can be part of power management system used in wireless sensor nodes, smart appliances or off-grid power systems.[1]

\section{LOSS MODELS OF DC-DC TOPOLOGIES}

Performance of heterogeneous parallel converter is evaluated by creating electrotechnical model of loss of individual parallel paths. It is best if convertor topologies forming parallel paths are diverse. Specialization of each path for unique conditions increase overall efficiency of the system. BuckBoost and Fly-back topologies are first candidates, due to differences by the use of simple inductor versus transformer [2]. Next evaluated is combination of two inductors and a capacitor in what is known as Zeta topology. Loss models are based on equations used by various circuit manufacturers [3] [4] [5] [6] [7] [8]. Flawless mathematical model of circuit loss would be more complex than is necessary for our purpose. Dispersion, degradation and temperature drift of component's properties make perfect model almost impossible. Models are limited to regard following losses: conduction loss on parasitic resistance, transistor gate switching and transistor linear mode. Evaluated operating frequencies are chosen in range from $10 \mathrm{kHz}$ to $250 \mathrm{kHz}$, so properties with insignificant impact within this range can be ignored, namely: loss on inductor core, transistor drain-source capacity, parasitic properties of wiring. This will increase loss on capacitor filtering output voltage, but its impact is also ignored as it is shared by all tested converters. Narrow frequency range allows model to consider frequency dependent properties as constants capacitor impedance, inductance value and parasitic resistance of inductors. Model assumes switching transistors operate synchronously without losses caused by imperfect timing. Due to all mentioned simplifications, created models are suitable for comparative study and not for precision tasks like control algorithm.

Important steps in calculation of loss model are :

- Calculating ciritical conduction current.

- Determining operating mode (continuous or discontinuous) by comparing critical and required output current.

- Calculating duty cycle for active mode.

- Calculating current flowing through each component. 


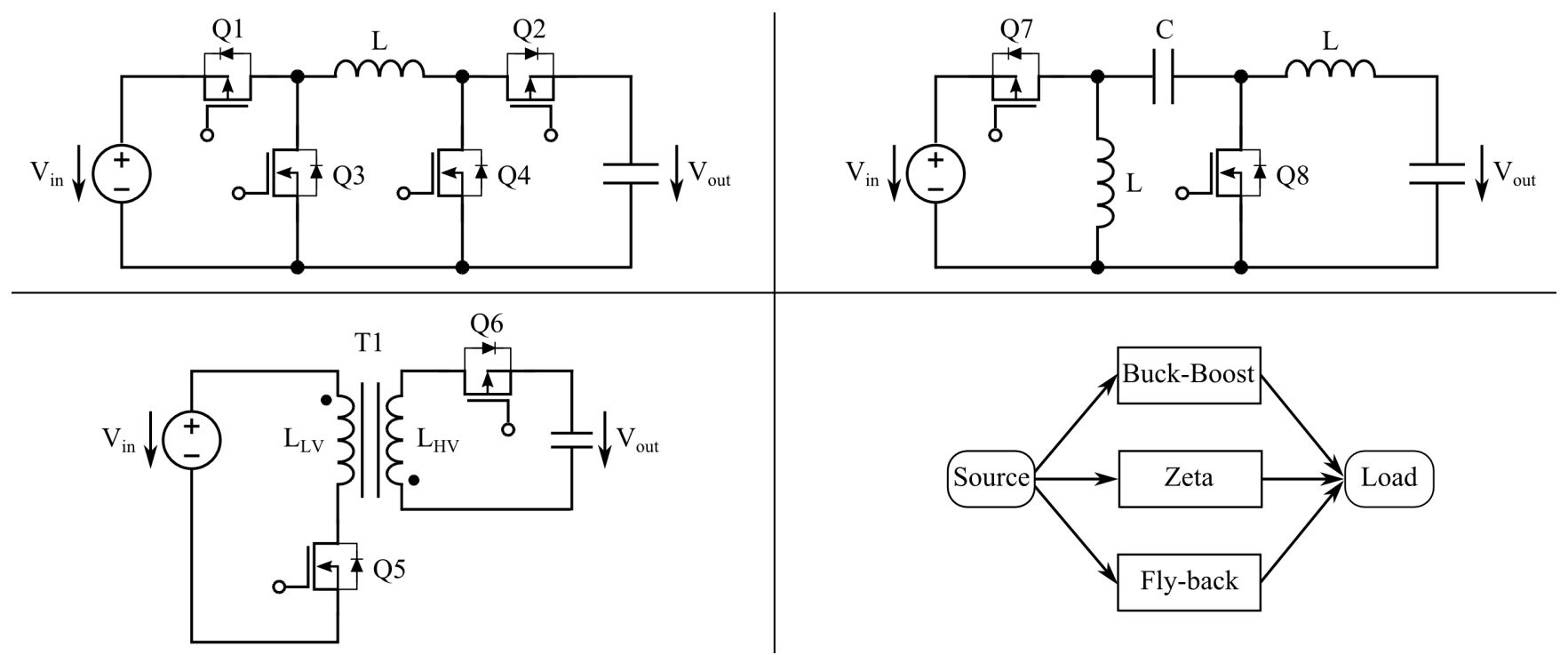

Fig. 1. Schematics of used converter circuits - (top left) four switch buck-boost, (top right) Zeta, (bottom left) Fly-back. Overview of formed multitopology converter is at bottom right.

TABLE I

LIST OF COMPONENTS USED FOR THE TESTING

\begin{tabular}{|l|c|}
\hline Schematic symbol & Component \\
\hline L & MCSDC0805-270KU \\
\hline Q3,Q4,Q5,Q8 (N-MOSFET) & SI2302CDS-T1-E3 \\
\hline Q1,Q2,Q6,Q7 (P-MOSFET) & SI2301CDS-TI-GE3 \\
\hline C & AVX 18125C475KAT2A \\
\hline \multirow{2}{*}{ T1 } & ratio $1: 100$ \\
\cline { 2 - 2 } & $\mathrm{R}_{L V}=0,1 \Omega \mathrm{R}_{H V}=3,3 \Omega$ \\
\cline { 2 - 2 } & $\mathrm{L}_{L V}=51 \mu \mathrm{H} \mathrm{L}_{H V}=5 \mathrm{mH}$ \\
\hline
\end{tabular}

Particular order of these steps depends on the form of equations describing the circuit. Other circuits may need extra steps specific to them. Model also requires parasitic values of used components. These were filled with typical values of common real world components listed in Table I. Transformer parameters were measured directly on specified part at fixed frequency $100 \mathrm{kHz}$, due to lack of documentation.
For desired output voltage $3.3 \mathrm{~V}$ and fixed switching frequency $100 \mathrm{kHz}$, these models form spaces shown in Figure 2. Combinations of these spaces at three different frequencies are in Figure 3. Both these figures show different characteristics of each converter topology. Buck-Boost is the only one that can pass input voltage directly to output. This is reflected by ridge in area where input and output voltages have the same value. Loss of this topology seems to increase the least with increased frequency, which may be caused by use of just one frequency dependant component: inductor. Zeta converter topology is expected to work the best at low frequencies for the same reason. Properties of fly-back converter are heavily dependent on inductance, resistance and ratio of transformer's windings. Space of fly-back converter also contains ridge with highest efficiency, however, its position is tied to switching frequency and is limited by operating voltages and current as well as component values. The ridge represents operation in critical conduction, the border between continuous and discontinuous conduction [9].
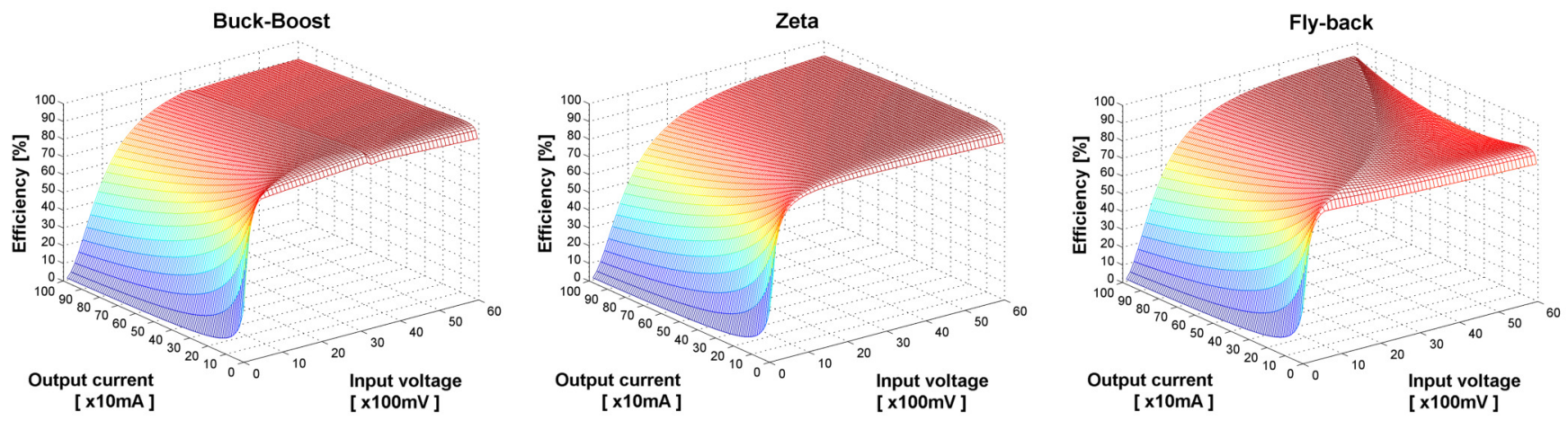

Fig. 2. Spaces of each topology at $\mathrm{V}_{\text {out }}=3,3 \mathrm{~V}$ and $\mathrm{f}=100 \mathrm{kHz}$ 

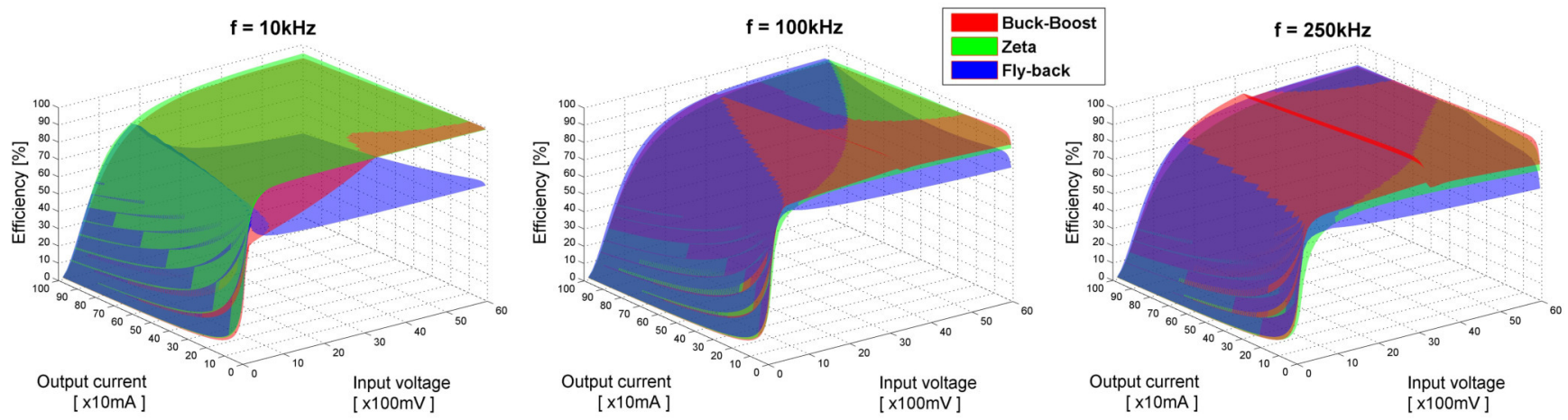

Fig. 3. Combined spaces at various frequencies at $\mathrm{V}_{\text {out }}=3,3 \mathrm{~V}$

\section{OPTIMIZATION METHODS}

\section{A. Exact methods}

In solving combinatorial problems we encounter the issue of how to ensure that the solution is quickly achievable and as accurate as possible. The first option are deterministic methods the steps of which are always repeated in the same order. Such methods, when properly implemented, offer the exact solution.

We used a method described in Algorithm 1 that searches through all solutions. It is a relatively time-consuming method that guarantees to find an optimal solution. At data extensive tasks, however, the growing complexity of computing and time-consumption takes effect. If we need to search through all solutions for only three variables ranging from 1 to 1000 , the number of iterations is about $10^{9}$. Addition of another variable will grow the number of iterations exponentially. The use of such a calculation method is purely for the verification purposes. The use in practice would be unsuitable, due to complexity. Example of this kind of method is "brute-force" or "branch and bound".

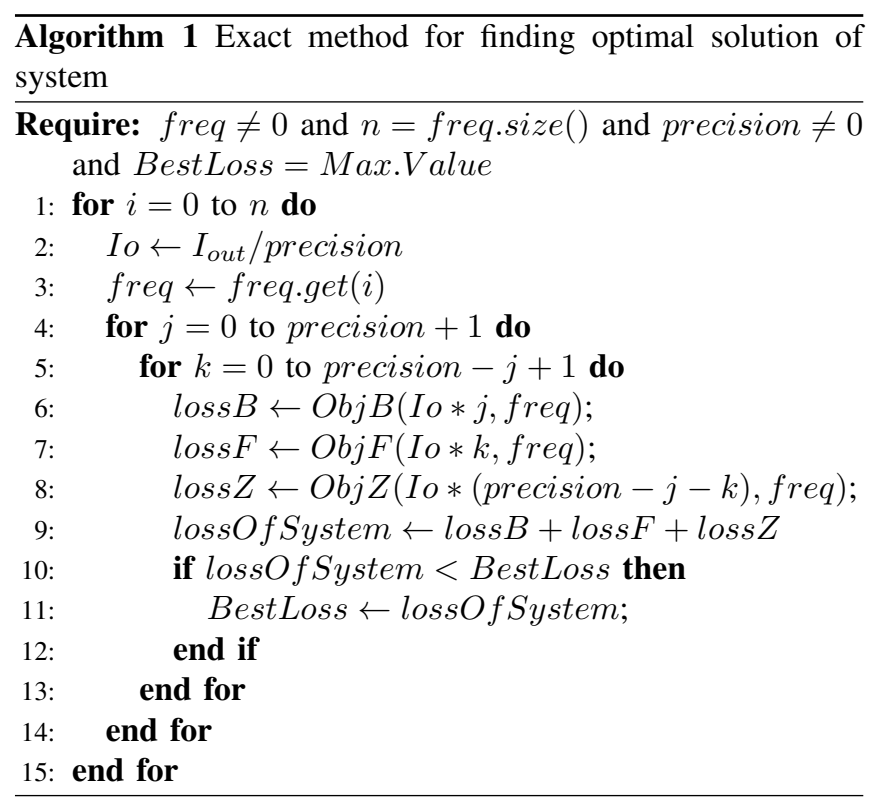

\section{B. Heuristic methods}

Since deterministic methods do not provide a suitable solution within the required time we need to implement methods that would provide us with such solution. One type of such methods are heuristic methods. These methods are based on experience and often provide a suitable solution in a relatively short amount of time, respectively a small number of iterations. These methods do not guarantee us to find optimal solutions.

Heuristic methods are frequently used for initial finding solutions to complicated methods, for example: metaheuristic. To find the base solution we used Monte Carlo method which in a very short time will provide us with a feasible solution for the next application [10]. The next step in finding a suitable solution for system was applying heuristic exchange. Since, in our case was no threat to get stuck in a local extreme, we could simplify the method. We allowed all valid transitions between solutions as well as the transition towards a worse value of objective function. However, we only impose those

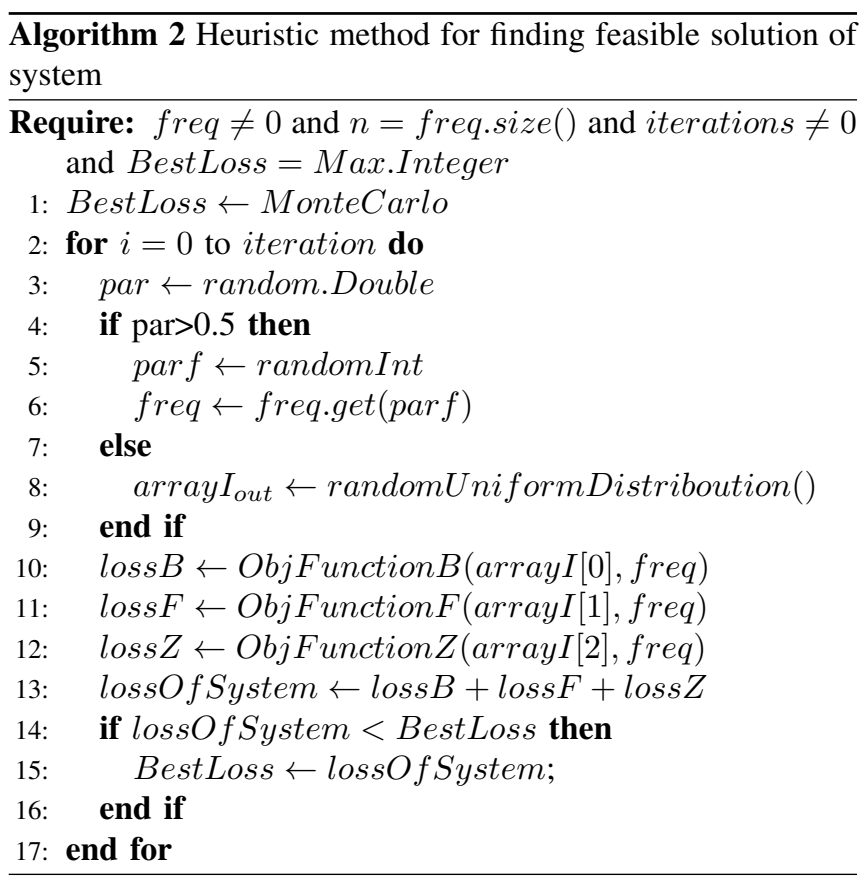


values into memory, for which the value of objective function gives a better solution. We have neglected all other solutions.

The method does not use the shutdown time, as is customary. Instead, it uses the number of iterations, which is introduced as a parameter and selected at the beginning. Authorized operation of this method is to exchange one for one. Since we are using global scanning, it means that we always change just one randomly selected parameter

Each algorithm that is not exact has a certain error rate depending on factors such as time, iteration number of inputs, and others. In this case, accuracy will result in the number of iterations. During our tests this method will be analyzed on various ranges of iterations starting on $10^{4}$ and ending with $10^{6}$ iterations. This is important for showing differences in results because the number of iterations greatly affects their accuracy.

Other algorithms such as genetic algorithm or taboo search algorithm show different ways how to obtain good solution. Genetic algorithm uses population of solutions and mixing them with steps like crossover and mutation. On other hand taboo search uses temporary prohibition after choosing different solutions to prevent from looping. Both approaches have their strong sides and their weak sides.

Not every heuristic method is suitable for solving a specific problem. Heuristic methods are built for specific problems even though the principles of the solution can be repeated. In contrast, metaheuristic methods are considered to be generally applicable methods of solution. It is recommended to use metaheuristic methods to solve problems where there is a risk of being deadlocked in local maximum or minimum [11].

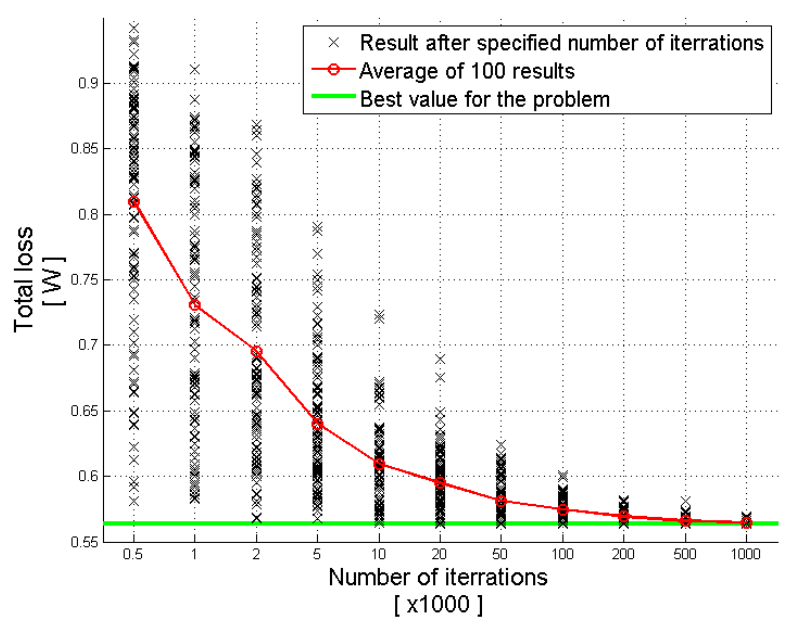

Fig. 4. Dependence of the total loss on the number of iterations.

\section{Simulation}

Performance of heterogeneous parallel converter is compared on scenarios where load requires constant supply of $2 \mathrm{~A}$ at $3.3 \mathrm{~V}$ from fully charged Li-po battery at $4.2 \mathrm{~V}$ or from Ni-MH battery at $1.2 \mathrm{~V}$. These values could reflect needs of wireless module inside battery powered sensor node. Simulation compares heterogeneous parallel converter with equal distribution to calculated optimal distribution. It is also compared to its homogeneous counterpart and non-parallel designs. Calculated values of the two scenarios at the most convenient frequencies are in Table III. These results show that the proposed heterogeneous parallel converter doesn't offer the lowest loss of them all, but rather low loss at wider operating conditions. This is illustrated better at the spaces created by the models. Due to all simplifications in the circuit model, absolute loss of real device would be higher. However, even distorted models allow to find best range for component values, which influence relative position of modeled spaces. Finding these values without the models would be impractical.

Optimization method used for load balancing is evaluated in Figure 4, which shows results of described heuristic method after specified range of number of iterations. To compensate for non-deterministic nature of heuristic method, every case is repeated 100 times. Optimal solution of the problem is calculated by exact "brute-force" method. Results of this simulation shows, that the number of iterations doesn't improve just average value of the solution, but also decreases dispersion of the results. Further improvements to optimization method can also decrease dispersion with minimal increase of computational requirements. One way of achieving this is to use pre-processed model data from other more powerful computer.

TABLE III

LOSS OF VARIOUS SIMULATED CONVERTERS

\begin{tabular}{|l|c|c|c|}
\hline \multirow{2}{*}{ System converters } & $\begin{array}{c}\mathrm{f} \\
{[\mathrm{kHz}]}\end{array}$ & $\begin{array}{c}\mathrm{V}_{\text {in }} \\
{[\mathrm{V}]}\end{array}$ & $\begin{array}{c}\text { System loss } \\
{[\mathrm{W}]}\end{array}$ \\
\hline Buck-Boost, Fly-back, Zeta \\
( Optimal distribution ) & 53 & 4.2 & 0.4457 \\
\cline { 2 - 3 } $\begin{array}{l}\text { Buck-Boost, Fly-back, Zeta } \\
\text { ( Equal distribution ) }\end{array}$ & 11 & 1.2 & 2.6731 \\
\cline { 2 - 3 } \multirow{2}{*}{ Buck-Boost,Buck-Boost, Buck-Boost } & 10 & 4.2 & 0.5147 \\
\cline { 2 - 3 } & 10 & 4.2 & 2.9626 \\
\cline { 2 - 3 } & 10 & 1.2 & 0.4901 \\
\multirow{2}{*}{ Fly-back,Fly-back, Fly-back } & 85 & 4.2 & 0.069 \\
\cline { 2 - 3 } & 10 & 1.2 & 2.4492 \\
\hline \multirow{2}{*}{ Zeta,Zeta, Zeta } & 10 & 4.2 & 0,4161 \\
\cline { 2 - 3 } & 10 & 1.2 & 3.1504 \\
\hline \multirow{2}{*}{ Buck-Boost } & 10 & 4.2 & 1.6267 \\
\cline { 2 - 3 } & 10 & 1.2 & 12.1465 \\
\hline \multirow{2}{*}{ Fly-back } & 26 & 4.2 & 0.8348 \\
\cline { 2 - 3 } & 10 & 1.2 & 7.2589 \\
\hline \multirow{2}{*}{ Zeta } & 10 & 4.2 & 0.8949 \\
\cline { 2 - 3 } & 10 & 1.2 & 8.0541 \\
\hline
\end{tabular}

TABLE II

NUMERICAL VALUES OF AVERAGE FUNCTION FROM FIGURE 4

\begin{tabular}{|c|c|c|c|c|c|c|c|c|c|c|c|}
\hline Iterations count & 500 & 1000 & 2000 & 5000 & 10000 & 20000 & 50000 & 100000 & 200000 & 500000 & 1000000 \\
\hline Arithmetic mean [W] & 0.81 & 0.7307 & 0.6956 & 0.64 & 0.6092 & 0.604 & 0.5814 & 0.5746 & 0.5693 & 0.565 & 0.5646 \\
\hline
\end{tabular}




\section{CONCLUSION}

Simplified circuit models suggest heterogeneous parallel DC-DC converter has potential to be more efficient than parallel converter with homogenous power paths. While homogeneous variant can achieve lower loss at specific conditions, heterogenous approach achieves low loss at wider operating conditions. Time characteristic of the source and load have significant impact on which of these approaches is more convenient. Load balancing of heterogeneous paths can be done by devices with low computational power at cost of precision. Use of described heuristic method leads to acceptable error within reasonable number of iterations. Optimization methods can be further improved by utilising pre-processed data from computer model.

\section{REFERENCES}

[1] Lingyin Zhao and Jinrong Qian, Texas Instruments, "DC-DC Power Conversions and System Design Considerations for Battery Operated System", January 2006

[2] S. Žák, P. Šarafín, P. Ševčík, "The multi-topology converter for the solar panel", in FedCSIS proceedings of the 2016 Federated conference on Computer science and information systems, 2016, pp. 1107-1110, ISBN 978-83-60910-92-7.
[3] TI Literature Number SLVA061, "Application Report Understanding Boost Power Stages in Switchmode PowerSupplies", March 1999

[4] TI Literature Number SLVA057, "Application Report Understanding Buck Power Stages in Switchmode PowerSupplies", March 1999

[5] E. Niculescu, D. Mioara-Purcaru, M. Niculescu, I. Purcaru, M. Maria, "A Simplified Steady-State Analysis of the PWM Zeta Converter", in Proceedings of the 13th WSEAS International Conference on Circuits,pp.108-113,ISSN:1790-5117, ISBN:978-960-474-096-3

[6] Anthony Fagnani, TI Literature Number SLVA559, "Application Report Isolated Continuous Conduction Mode Flyback Using the TPS55340", January 2013

[7] Mohammad Kamil, Microchip Technology Inc., "AN1114 - Switch Mode Power Supply (SMPS) Topologies (Part I)", 2007

[8] Antonio Bersani, Microchip Technology Inc., "AN1207 - Switch Mode Power Supply (SMPS) Topologies (Part II)", 2008

[9] G. Spiazzi, D. Tagliavia and S.Spampinato, "DC-DC flyback converters in the critical conduction mode: a Re-examination" in Proc. IEEE IAS Conf., vol. 4, 2000, pp. 2426-2432.

[10] Szabo, J., "Comparison of Methods for Generating Initial Solution for Simulated Annealing", CERES Central European Researchers Journal 2, Issue 1, 37-41, 2016

[11] Amin Alqudah, Ahmad Malkawi, Abdullah Alwadie, "Adaptive Control of DC-DC Converter Using Simulated Annealing Optimization Method", in Journal of Signal and Information Processing, 2014, 5, pp. 198-207 